\title{
Cervical Adenocarcinoma Admixed with Neuroendocrine Carcinoma
}

National Cancer Institute

\section{Source}

National Cancer Institute. Cervical Adenocarcinoma Admixed with Neuroendocrine

Carcinoma. NCl Thesaurus. Code C127915.

A carcinoma that arises from the cervix and is characterized by the presence of a cervical adenocarcinoma variant and a neuroendocrine carcinoma component. 